J. Clin. Chem. Clin. Biochem.

Vol. 15, 1977, pp. 367-369

\title{
Eine schnelle teilautomatische Abtrennung der Katecholamine aus Urin
}

Von H. H. Bussemas, Chr. Lippmann und G. Schwedt

Institut für Arbeitsphysiologie (Geschäftsführender Institutsleiter: Prof. Dr. H. G. Wenzel) an der Universität Dortmund

(Eingegangen am 9. Dezember 1976)

Zusammenfassung: Die teilautomatische Abtrennung der Katecholamine Adrenalin und Noradrenalin beruht auf der Adsorption an Aluminiumoxid bei pH 8,4 und anschließender Desorption mit 0,05 mol/1 Perchlorsäure im Batchverfahren. Die einzelnen Teilschritte wurden untersucht und optimiert. Die Vorteile des Verfahrens gegenüber der manuellen Säulentechnik sind verringerter Zeit- und Personalbedarf, verminderte Gefahr des Einschleppens von Störsubstanzen und exakte Einhaltung der zeitlichen Bedingungen.

Die Wiederfindung beträgt für Adrenalin $87,3 \pm 4,1 \%$ und für Noradrenalin $71,9 \pm 5,9 \%$.

\section{A rapid semiautomatic separation of urinary catecholamines}

Summary: The semiautomatic separation of the catecholamines, adrenaline and noradrenaline, is based on their adsorption onto aluminium oxide at $\mathrm{pH} 8.4$, followed by batch elution with $0.05 \mathrm{~mol} / 1$ perchloric acid. The separate steps of the operation were investigated and optimised. Advantages of the method, compared with the manual column technique, are decreased time of operation, less involvement of personel, less danger of carry-over of interfering substances, and an exact standardisation of the processing time.

The recovery was $87.3 \pm 4.1 \%$ for adrenaline, and $71.9 \pm 5.9 \%$ for noradrenaline.

\section{Einführung}

Von den möglichen Abtrennungsverfähren für die Katecholamine Adrenalin und Noradrenalin aus Urin hat sich die Adsorption an Aluminiumoxid bewährt und in der Prạxis durchgesetzt (1). Das von uns bisher angewendete Säulenverfahren (2) ist für hohe Probenzahlen zu arbeitsund zeitintensiv und läßt sich im Hinblick auf eine schnellere Durchführung nịcht wesentlich verbessern; hingegen bietet die Abtrennung im Batch-Verfahren günstige Möglichkeiten für eine Teilautomatisierung.

\section{Material und Methoden}

\section{Untersuchungsmaterial}

Harn wird sofort nach Gewinnung mit $5 \mathrm{~mol} / 1$ Schwefelsäure auf pH 1-2 angesäuert und kühl aufbewahrt: bis 1 Woche bei $+4{ }^{\circ} \mathrm{C}$, längere Zeit bei $-20^{\circ} \mathrm{C}$.

\section{Geräte}

Multi-D̈osimat-Titrierstand E 415 (Mețrohm, Herisau) pH-Meter E 388 (Metrohm)

Reagenzglas-Rotierer (Eigenbau in Anlehnung an Cordes (pertsönl. Mitt.):
12 Reagenzgläser im Ständer rotieren mit $60 \mathrm{U} / \mathrm{min}$ um ihre vertikale Achse

10 ml-Reagenzgläser mit Ringmarke und NS 12,5 - Polyethylenstopfen

Vibro-Fix (Janke \& Kunkel KG, Staufen)

Wasserstrahlpumpe

Schüttelmaschine

\section{Reagenzien und Lösungen}

Aluminiumoxid sauer (Woelm)

Phosphat-Lösung: Dinatriumhydrogenphosphat-2-hydrat p. A. (Merck) $5 \mathrm{~g} / 1$ in Wasser, $\mathrm{pH} 8,4$

EDTA-Lösung: Titriplex III p. A. (Merck) $50 \mathrm{~g} / 1$ in Wasser Natronlauge: Natriumhydroxid p. A. (Merck) $80 \mathrm{~g} / 1$ in Wasser Perchlor säure: Perchlorsäure $700 \mathrm{~g} / \mathrm{kg}$ p. A. (Merck) $0,05 \mathrm{~mol} / 1$ Phenolphthalein-Lösung: Phenolphthalein Indikator (Merck) $1 \mathrm{~g} / \mathrm{l}$ in Ethanol

\section{Durchführung}

Feine Anteile des Aluminiumoxids durch wiederholtes Suspendieren in bidest. Wasser (etwa $10 \mathrm{X}$ ) entfernen, im Trockenschrank etwa eine Woche bei $40^{\circ} \mathrm{C}$ trocknen.

$0,5 \mathrm{~g} \mathrm{Al}_{2} \mathrm{O}_{3}$ in Reagenzglas einwägen

$+5 \mathrm{ml}$ Urinprobe

+2 ml EDTA-Lösung

$+0,1 \mathrm{ml}$ Phenolphthalein-Lösung

+ Natronlauge bis zur deutlichen Rotfärbung (pH 8,4) titrieren, dabei kräftig durchmischen (Vibro-Fix)

+ Phosphat-Lösung bis zur Marke auffüllen 
5 min rotieren lassen (Adsorption), überstehende Lösung absaugen

+ etwa $5 \mathrm{ml}$ Phosphatlösung

5 min rotieren lassen, überstehende Lösung absaugen

+ etwa $5 \mathrm{ml}$ bidest. Wasser

5 min rotieren lassen, überstehende Lösung absaugen

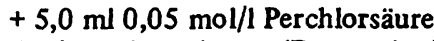

$5 \mathrm{~min}$ rotieren lassen (Desorption).

Anstelle des Rotierers kann auch eine Schüttelmaschine benutzt werden.

Die fluorimetrische Bestimmung wurde mit einem automatischen Analysensystem (3) in Anlehnung an Wisser (4) nach der Trihydroxyindol-Methode vorgenommen.

Die einzelnen Teilschritte der Abtrennung wurden systematisch untersucht. Um die optimalen Bedingungen zu ermitteln, wurden für jede Veränderung fünfmal $5 \mathrm{ml}$ angesäuerter Urinprobe eingesetzt (Ergebnisse siehe Tab. 1).

Tab. 1. Ergebnisse $(\bar{x} \pm s)$ aus den Optimierungsversuchen $(n=5)$.

\begin{tabular}{|c|c|c|c|c|}
\hline \multirow{2}{*}{$\begin{array}{l}\text { Bedingung } \\
\text { Waschen mit heißer } \\
\text { Säure, Trocknen bei } \\
200^{\circ} \mathrm{C}\end{array}$} & \multicolumn{2}{|c|}{$\begin{array}{l}\text { Adrenalin } \\
\text { [nmol/1] }\end{array}$} & \multicolumn{2}{|c|}{$\begin{array}{l}\text { Noradrenalin } \\
\text { [nmol/1] }\end{array}$} \\
\hline & 98,3 & $\pm 2,7$ & 288,4 & $\pm 12,4$ \\
\hline $\begin{array}{l}\text { Waschen mit Wasser, } \\
\text { Trocknen bei } 40^{\circ} \mathrm{C}\end{array}$ & 101,5 & $\pm 2,2$ & 279,6 & $\pm 1,8$ \\
\hline $\begin{array}{l}\text { pH-Wert-Einstellung } \\
\text { mit Glaselektrode } \\
\text { (Becherglas) }\end{array}$ & 52,4 & $\pm 5,5$ & 287,2 & $\pm 13,0$ \\
\hline $\begin{array}{l}\text { mit Indikator } \\
\text { (Reagenzglas) }\end{array}$ & 59,5 & $\pm 12,6$ & 277,2 & $\pm 13,6$ \\
\hline \multicolumn{5}{|l|}{ Adsorption } \\
\hline $\begin{array}{l}\text { ohne Kühlung } \\
\text { mit Kühlung }\end{array}$ & $\begin{array}{l}36,0 \\
32,8\end{array}$ & $\begin{array}{l} \pm \quad 4,9 \\
\pm \quad 3,3\end{array}$ & $\begin{array}{r}87,5 \\
101,1\end{array}$ & $\begin{array}{l} \pm 3,0 \\
\pm 8,3\end{array}$ \\
\hline \multicolumn{5}{|l|}{ Wiederfindungsrate } \\
\hline $\begin{array}{l}\text { mit } 0,2 \mathrm{~mol} / 1 \text { Essigsäure } \\
\text { mit } 0,05 \mathrm{~mol} / 1 \mathrm{HClO}_{4}\end{array}$ & $\begin{array}{r}0,76 \\
0,87\end{array}$ & $\begin{array}{l}1 \pm 0,060 \\
3 \pm 0,041\end{array}$ & $\begin{array}{l}0,66 \\
0,71\end{array}$ & $\begin{array}{l} \pm 0,042 \\
\pm \quad 0,059\end{array}$ \\
\hline
\end{tabular}

\section{Ergebnisse und Diskussion}

\section{Vorbehandlung des Aluminiumoxids}

Gebräuchlich ist das Waschen mit heißer Säure und Trocknen bei $200^{\circ} \mathrm{C}$. Das Waschen mit Wasser und Trocknen bei $40^{\circ} \mathrm{C}$ (Tab. 1) reicht jedoch aus.

\section{Einstellen des $\mathrm{pH}$-Wertes}

Um Überführungsschritte möglichst $\mathrm{zu}$ vermeiden, sollte das Einstellen des $\mathrm{pH}$-Wertes direkt im Reagenzglas vorgenommen werden. Die Verwendung einer normalen Glaselektrode wie beim Säulenverfahren ist dabei nicht möglich. Wie von Merrils (5) beschrieben, läßt sich zur Einstellung des pH-Werts von 8,3-8,5 Phenolphthalein als Indikator einsetzen (Tab. 1).

\section{Adsorption}

Als ausreichende Menge Aluminiumoxid wurde 0,5 g $\mathrm{Al}_{2} \mathrm{O}_{3}$ für $5 \mathrm{ml}$ Urin ermittelt (s. Abb. 1). Nach $5 \mathrm{~min}$

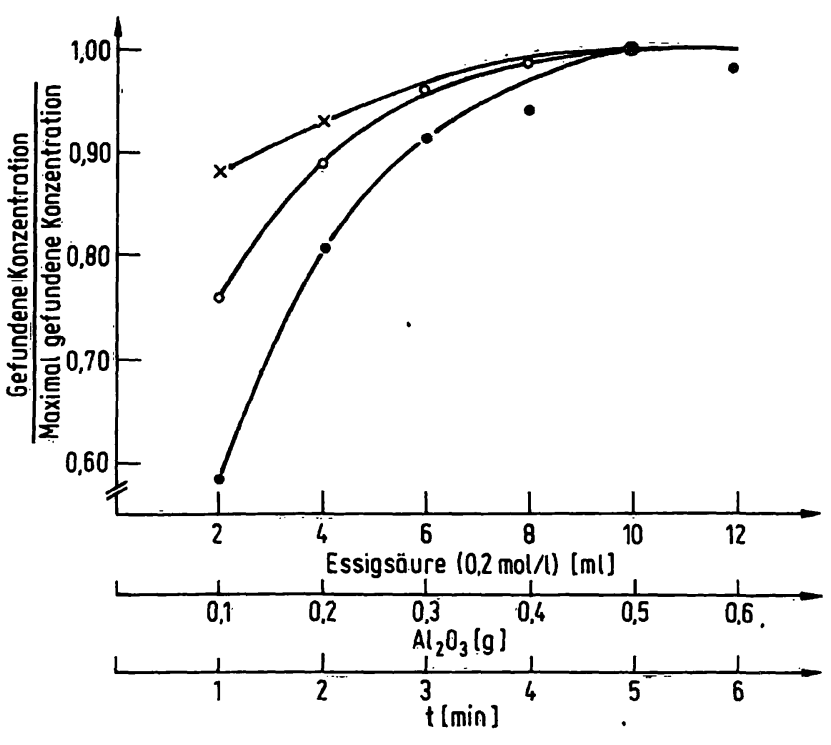

Abb. 1. Optimierung der Methode.

Gefundene relative Konzentration ( $\bar{x}$ ) von Adrenalin und Noradrenalin im Reaktionsansatz (5 ml Urin) in

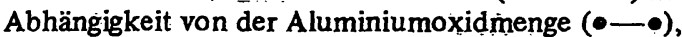
der Adsorptionszeit $(x-x)$ und vom Desorptionsvolumen $(0-0)$.

ist die Adsorption abgeschlossen (s. Abb. 1). Kühlung nach 1.c. (2) (siehe Tab. 1) und stabilisierende Zusätze wie Ascorbinsäure (5) oder Natriummetabisulfit (6) verbessem weder die Wiederfindung noch die Reproduzierbarkeit des Verfahrens.

\section{Waschen des Aluminiumoxids nach der Adsorption}

Das Waschen des Aluminiumoxids nach der Adsorption kann auf das angegebene Maß beschränkt werden, ohne daß Störungen auftreten.

\section{Desorption}

Zur Ermittlung der für die Desorption am besten geeigneten Säure wurden Essigsäure (Bereich 0,2-1,0 mol/1) und Perchlorsäure (Bereich 0,05-0,2 mol/1) eingesetzt. In Übereinstimmung mit Mabry (7) fanden wir 0,05 mol/1 Perchlorsäure in bezug auf Wiederfindung und Reproduzierbarkeit als optimal (Tab. 1). Aus dieser Lösung kann zudem die fluorimetrische Bestimmung unmittelbar, d. h. ohne Abtrennung des Aluminiumoxids und Einstellung des pH-Werts, erfolgen.

Da einerseits die Wiederfindung mit abnehmendem Desorptionsvolumen sinkt, andererseits ạber mit größeren Desorptionsvolumina die Fluoreszenzintensitäten bei der Messung durch die Verdünnung kleiner werden, ermittelten wir als Optimum $5 \mathrm{ml}$ der verdünnten Säure (siehe Abb. 1).

\section{Vorteile der Abtrennungsmethode}

1. Von einer Person können 60 Abtrennungen pro Tag ausgeführt werden. Damit verrịingert sich der Zeit- und 
Personalbedarf auf etwa ein Drittel, verglichen mit dem bisherigen Säulenverfahren.

2. Die gesamte Abtrennung findet in einem einzigen Reagenzglas statt. Die Gefahr des Einschleppens von Störsubstanzen ist beträchtlich vermindert.

\section{Literatur}

1. Schwedt, G. (1976), in Tagungsbericht der Deutschen Gesellschaft für Arbeitsmedizin e. V., S. 249-252, Gentner Verlag, Stuttgart.

2. Schwedt, G. (1974), Clin. Chim: Acta 51, 247-256.

3. Schwedt, G. \& Bussemas, H. H. (1975), Z. Anal. Chem. 276, $55-59$.
3. Durch die Teilmechanisierung können die zeitlichen Bedingungen der Abtrennung exakter als im Säulenverfahren eingehalten werden (2).

4. Wisser, H. (1970), diese Z. 8, 637-648.

5. Merrils, R. J. (1963), Analyt. Biochem. 6, 272-282

6. Anton, A. H. \& Sayre, D. F. (1962), J. Pharmacol. Exper.

Therap. (Baltimore) 138, 360-375.

7. Mabry, C. C. \& Warth, P. W. (1969), Am. J. Clin. Pathol. 52, 57-68.

H. H. Bussemas

Institut für Arbeitsphysiologie

Ardeystraße 67

D-4600 Dortmund 


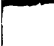

